\title{
Phenomenological description of a spin chain system with geometrical frustration of couplings
}

\author{
A.A. Zvyagin \\ Max-Planck-Institut für Physik komplexer Systeme, 38 Nöthnitzer Str., Dresden 01187, Germany \\ B. Verkin Institute for Low Temperature Physics and Engeneering of the National Academy of Sciences of Ukraine \\ 47 Nauky Ave., Kharkiv 61103, Ukraine \\ E-mail: zvyagin@ilt.kharkov.ua
}

Received April 18, 2017, published online September 25, 2017

\begin{abstract}
The phenomenological model, using which we can in a relatively simple way calculate many magnetic, thermodynamic and dynamic characteristics of the spin chain material with the geometrical frustration of spin-spin couplings is proposed. The results of theoretical calculations well reproduce observed details of the low-temperature behavior of the magnetization, magnetic susceptibility, specific heat, magneto-acoustic characteristics, and some dynamical properties. In particular, the model permits to explain the double peak structure of the temperature dependencies of the magnetic susceptibility, specific heat, and the renormalization of the sound velocity, and can explain several features of the ESR frequency-field diagram.
\end{abstract}

PACS: 75.10.Jm Quantized spin models, including quantum spin frustration;

75.10.Pq Spin chain models;

75.40.Cx Static properties;

75.40.Jb Dynamic properties.

Keywords: spin chain system, magnetic susceptibility, specific heat, sound velocity.

Quantum spin liquids are characterized by strong spinspin couplings. Emergent excitations of quantum spin liquids are fractionalized quasiparticles. Among the large variety of magnetic materials low dimensional spin systems and systems with frustration are known to demonstrate properties of quantum spin liquids [1]. Frustrated bonds, as well as quantum fluctuations, enhanced in lowdimensional spin systems, often tend to destroy the magnetic ordering in quantum spin liquids. The progress in preparation of magnetic materials with well defined onedimensional (1d) subsystems has motivated the interest in their studies during last years. Another reason for the investigation of properties of quasi-1d spin systems is the relatively rare possibility of comparison of the data of experiments with the results of non-perturbative theories for many-body models [2]. According to the Mermin-Wagner theorem [3], totally 1d spin systems with isotropic spinspin interactions cannot reveal any magnetic ordering at nonzero temperatures. However, for quasi-1d spin systems, which $1 \mathrm{~d}$ subsystems have gapless low-lying excitations, the magnetization, magnetic susceptibility, specific heat, etc. often manifest peculiarities, characteristic for phase transitions to magnetically ordered states. Frustrated spin systems also manifest peculiar properties [4], which distinguish them from standard magnetically ordered systems and from ordinary paramagnets [5]. The system is called frustrated if one cannot minimize its total energy (the macroscopic state) by minimizing the energy of each pair involving into the interaction. The frustration often produces a high level of degeneracy of eigenstates. It is very difficult to construct theories to explain the behavior of frustrated spin systems, because the absence of the well-defined nondegenerate ground state does not permit to use the powerful methods of the magnetism theory, as the mean fieldlike approximations, or the spin wave approach. Therefore, approximate models, even "exotic" ones, the monopole theory for spin ices [6] being the prime example, are very useful for the description of spin systems with geometrical frustration.

For many quasi-1d spin systems and systems with the geometrical frustration, in which (maximal) exchange parameters along the distinguished direction are relatively small $(\sim 1-40 \mathrm{~K})$, the features of their behavior can be governed by the external magnetic field. The latter can induce quantum phase transitions, which manifestations at nonzero, however low temperatures can be observed. In 
low-temperature experiments high values of the magnetic field (about $20 \mathrm{~T}$ for stationary fields and about $60 \mathrm{~T}$ for pulse fields) can be used nowadays. Therefore, for many quasi-1d spin systems it is possible to investigate experimentally how the external magnetic field affects their properties. It is important for experimentalists, who study magnetic properties of frustrated low-dimensional spin systems, to use relatively simple theories, for comparison of their results with the prediction of those theories.

The observation of the $1 / 3$ magnetization plateau [7] in the low-temperature magnetization behavior of the natural mineral azurite $\mathrm{Cu}_{3}\left(\mathrm{CO}_{3}\right)_{2}(\mathrm{OH})_{2}$ has attracted the interest of researchers to that compound. Similar magnetization plateaux were observed in many frustrated spin systems [8], hence those plateaux often serve as the "benchmark" of frustrated magnetic materials. The structure of azurite (a monoclinic crystal structure with the space group $P 2_{1} / c$, where the magnetic ions $\mathrm{Cu}^{2+}$ are arranged in chains build of diamond-shaped units, running along $b$ direction of the crystal) implies the quasi-1d spin system with weakly coupled diamond spin chains (with mostly antiferromagnetic spin-spin interactions [9]). Formally diamond spin chains have the geometric frustration [9]. However, the characteristics of azurite do not show a high level of degeneracy, hence, the weak inter-chain coupling produces the magnetic ordering at low temperatures $T_{N} \sim 1.85 \mathrm{~K}$ [10], while some other features of the low-temperature magnetic behavior are characteristic for geometrically frustrated spin systems. The inelastic neutron scattering did not observe a strong dispersion perpendicular to the chain direction [10] even at $T<T_{N}$. Therefore, azurite at low enough temperatures, however for $T>T_{N}$, can be considered as the good representative of quantum spin liquids having, on the one hand, a strong spin-spin coupling along chains, and, on the other hand, frustrated spin-spin bonds, together with the fractional emergent excitations, see below.

There were many attempts to describe theoretically the properties of azurite, see, e.g., [7,10-14], etc. However, most of them either explain analytically only few features of the behavior of azurite characteristics, or use numerical calculations like the exact diagonalization, or the DMRG. The disadvantage of the latter is in the necessity of the production of new calculations with non-clear results to obtain results for a new set of parameters, and hence, long times of computer calculations. This is why, the objection of the present study is to construct an analytic theory (convenient for comparison with experiments), which can be used to describe many features of the behavior of azurite from the same grounds. In this contribution we propose the relatively simple analytic approach, which models such a quasi-1d frustrated spin system, revealing many properties of quantum spin liquids. The results of our approach manifest a very good agreement with the data of experiments related to magnetic, thermodynamic, and dynamic properties of azurite.
Let us start with the description of the magnetic field dependence of the magnetization of azurite. It manifests one of the most interesting features, the magnetization plateau at approximately $1 / 3$ of the total magnetization in saturation $M_{0}$ [7]. The magnetization grows with the external magnetic field $H$ for $H<H_{1}$, where $H_{1}$ is the first critical value of the field, and for $H_{2}<H<H_{s}$, where $H_{2}$ is the second critical value, and $H_{s}$ is the value of the field, at which the magnetization is saturated. On the other hand, the magnetization is almost constant as a function of the field $\left(\sim(1 / 3) M_{0}\right.$ for $H_{1}<H<H_{2}$ and $\sim M_{0}$ for $H>H_{s}$ ) at low temperatures. The magnetization of the $1 \mathrm{~d}$ spin system can have magnetization plateaux according to the generalization of the Lieb-Schulz-Mattis theorem [2,15,16], including the case of three critical values of the field. For example, three critical values of the magnetic field is the characteristic feature for the behavior of the trimerized exactly solvable quantum spin chain $[2,17]$. The Hamiltonian of the exactly solvable trimerized spin $1 / 2$ chain is $[2,17]$

$$
\begin{gathered}
\mathcal{H}_{3}=(1 / 2) \sum_{n}\left[\left(J_{0} S_{n, 0}^{+} S_{n, 1}^{-}+J_{1} S_{n, 1}^{+} S_{n, 2}^{-}+\right.\right. \\
\left.+J_{2} S_{n, 2}^{+} S_{n+1,0}^{-}+\text {H.c. }\right)- \\
\left.-2 \mu_{B} H\left(g_{0} S_{n, 0}^{Z}+g_{1} S_{n, 1}^{Z}+g_{2} S_{n, 2}^{Z}\right)\right]
\end{gathered}
$$

where $J_{0,1,2}$ determine the values of the exchange integrals between neighboring spins along the chain, $S_{n, 0,1,2}^{ \pm} \equiv$ $\equiv S_{n, 0,1,2}^{X} \pm i S_{n, 0,1,2}^{y}, g_{0,1,2}$ are the values of the $g$-factor for each of three non-equivalent magnetic centers, and $\mu_{B}$ is the Bohr magneton. After the generalized Jordan-Wigner transformation, the Fourier transform, and the unitary transformation [2,17] the Hamiltonian can be presented as the sum of three diagonal quadratic forms of spinless fermion operators

$$
\mathcal{H}_{3}=\sum_{j=0}^{2} \sum_{k} \varepsilon_{k, j} b_{k, j}^{\dagger} b_{k, j}-\left[L \mu_{B} H\left(g_{0}+g_{1}+g_{2}\right) / 2\right],
$$

where $b_{k, j}\left(b_{k, j}^{\dagger}\right)$ destroys (creates) the spinless fermion of the branch $j=0,1,2$ with the quasimomentum $k$, and $3 L$ is the length of the chain (we use units in which the inter-cite distance is unity). For the simplest case $g_{0}=g_{1}=g_{2}=g$ the dispersion relations can be presented as

$$
\varepsilon_{k, j}=g \mu_{B} H-\frac{2 \sqrt{J_{0}^{2}+J_{1}^{2}+J_{2}^{2}}}{3} \cos \left(\alpha+\frac{2 \pi j}{3}\right),
$$

where $j=1,2,3$, and

$$
\alpha=\frac{1}{3} \arccos \left[-\frac{3 \sqrt{3} J_{0} J_{1} J_{2} \cos (k)}{\left(J_{0}^{2}+J_{1}^{2}+J_{2}^{2}\right)^{3 / 2}}\right] .
$$

One can see that all thermodynamic properties of the exactly solvable trimerized spin-1/2 chain are described by 
three non-interacting fermionic Hamiltonians, which parameters depend on the $g$-factors and exchange integrals of the spin chain, and on the value of the external magnetic field. The ground state for any fermion system is organized by the filling of all states with negative energies (the Fermi sea). Excitations are related either to the creation of particles with positive energies with respect to the Fermi energy, or to the creation of holes below the Fermi energy. It is easy to see that for $H=0$ one branch of the spectrum $\varepsilon_{k, 0,1,2}$ is gapless, and two others are gapped. The magnetic field (we consider $H \geq 0$ here and below) plays the role of the effective chemical potential for each branch. With the growth of the value of the magnetic field $H$, the Fermi level of the gapless branch goes down, so that above the critical value of the field $H_{1}$ the gapless branch becomes gapped. In the region $H_{1} \leq H \leq H_{2}$ all branches are gapped. Then, when the value of the magnetic field continues to grow, at the critical value $\mathrm{H}_{2}$ the second branch becomes gapless. Finally for $H>H_{S}$ the second branch again becomes gapped. The third branch of the spectrum is gapped for any value of the field $H$. For $H>H_{s}$ the total ground state magnetization of the trimerized exactly solvable spin $1 / 2$ chain (normalized by $3 L g \mu_{B}$ ) has the value 1 , while for $H_{1}<H<H_{2}$ it is $1 / 3$ (for those values of the field all branches are gapped). The ground state magnetization grows with $H$ only for the values of the field $0 \leq H \leq H_{1}$ and $H_{2} \leq H \leq H_{3}$, where some of the eigenstates have negative energies. Thus, the behavior of the magnetization of the trimerized exactly solvable spin chain is reminiscent of the one for azurite [7].

We can see that at least three non-equivalent magnetic centers are present in azurite. Moreover, according to the neutron scattering, the dispersion exists mostly along one direction in azurite [10], at least at intermediate temperatures $T \geq T_{N}$. This is why, to describe thermodynamic characteristics of azurite we propose to use the phenomenological model with the simple Hamiltonian

$$
\mathcal{H}_{p}=\sum_{j=1}^{3} \sum_{k} \varepsilon_{k, j}\left[b_{k, j}^{\dagger} b_{k, j}-(1 / 2)\right],
$$

where the energies are

$$
\begin{gathered}
\varepsilon_{k, 1}=g_{1} \mu_{B} H-J_{A} \cos (k), \\
\varepsilon_{k, 2,3}=g_{2} \mu_{B} H \pm \frac{1}{2} \sqrt{J_{B}^{2}+J_{C}^{2}+2 J_{B} J_{C} \cos k}
\end{gathered}
$$

Here $J_{A, B, C}$ are phenomenological exchange constants, and $g_{1,2}$ are effective $g$-factors to be determined from the fitting of the characteristics of the model to the values, obtained from the experiments on azurite. The properties of the phenomenological model are similar to the exactly solvable trimerized model. There are three branches of fermion excitations, and the external magnetic field plays the role of the effective chemical potential for fermions in each branch. The illustration of the behavior of the spectra of the model is shown in Fig. 1. The parameters are taken from the fitting of the experimental results for the magnetization of azurite for $H$ directed perpendicular to the axis $b$, see below. In fact, this phenomenological model describes the behavior of two independent spin-1/2 chains: the homogeneous chain and the dimerized one [2], both with the XXO magnetic anisotropy. Notice that it is only the phenomenological model. We cannot exactly derive this model from the microscopical structure of azurite like the approach of Refs. 10-14. However, the estimations of the values of the parameters of the phenomenological model, see below, can be related to the estimations of the exchange integrals of azurite using similar considerations, as in Refs. 7, 10-14. Also, the magnetic anisotropy of the inter-ion type, present in $X X 0$ spin chains with the excitation spectra Eqs. (5), is not related to the real magnetic anisotropy of azurite. Here we use just the phenomenological theory, similar to the approach of Ref. 18, where the strongly anisotropic $X X Z$ spin-1/2 chain model together with the fixed value of the applied effective field was successfully used to describe the properties of the isotropic (or weakly anisotropic) two-leg spin ladder. The dispersion in our model is along one space direction, which also in agreement with the behavior of azurite [10]. The phenomenological model is reminiscent of (but not equal to) the monomer-dimer model $[9,10]$. The main difference is in the presence of the third branch of excitations, totally absent for the monomer-dimer model.

Let us now fit the behavior of the low-temperature magnetization of azurite [7] using our phenomenological model. It gives for the magnetization per site

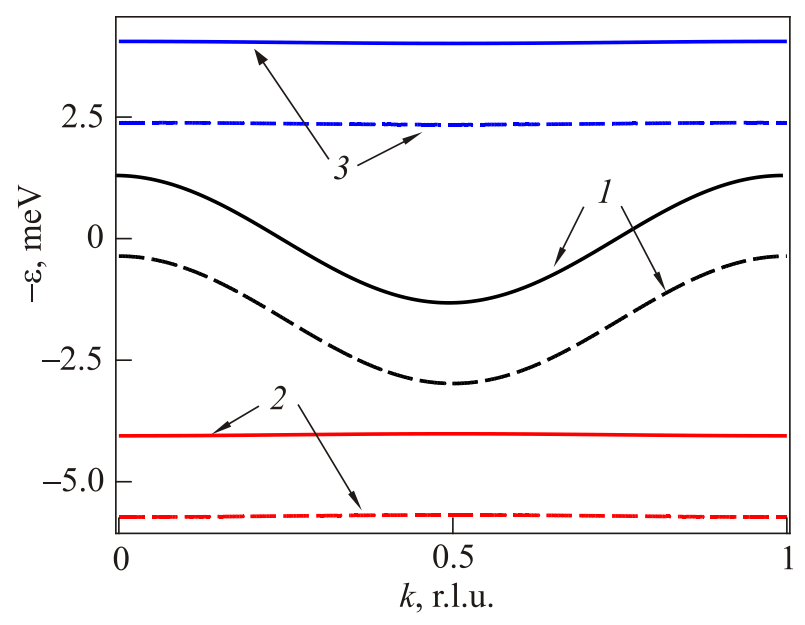

Fig. 1. (Color online) Three branches of eigenvalues (black (1), red (2) and blue (3) lines) of the phenomenological model with $J_{A}=$ $=1.31 \mathrm{meV}, J_{B}=0.42 \mathrm{meV}$, and $J_{C}=8.07 \mathrm{meV}$. Solid lines: $H=0$; dashed lines $g \mu_{B} H=14 \mathrm{~T}$. 


$$
\frac{M}{M_{0}}=\frac{1}{6 \pi} \sum_{j=1}^{3} \int_{0}^{\pi} d k \tanh \left(\frac{\varepsilon_{k, j}}{2 k_{B} T}\right),
$$

where $k_{B}$ is the Boltzmann constant, and $M_{0}$ is the maximal value of the magnetization. Figure 2 shows the result of such a fit for the magnetic field directed perpendicular to the direction of the chains $b$. We used the values for the exchange integrals $\left(J_{A}^{p} / g \mu_{B}\right)=11 \mathrm{~T}, \quad\left(J_{B}^{p} / g \mu_{B}\right)=3.5 \mathrm{~T}, \quad$ and $\left(J_{C}^{p} / g \mu_{B}\right)=64.5 \mathrm{~T}$ (the index $p$ signals about the orientation of the external magnetic field perpendicular to the chain direction) with the effective average $g$-factor for azurite taken from the ESR measurements $g_{1}=g_{2}=g=2.06$ [19] at the values of the temperature $T=0.08 \mathrm{~K}, T=1.3 \mathrm{~K}$, and $T=4.2 \mathrm{~K}$. One can see the excellent agreement of the data of experiment with the results of calculations for the phenomenological model. Notice that the magnetic ordering at $T=T_{N}$ almost does not affect the higher-energy branches of the phenomenological model (their contribution to the ordering is exponentially small). On the other hand, the difference in the behavior of the magnetization of the magnetically ordered antiferromagnet (see below) for $H \leq H_{1}$ does not differ drastically from the behavior of the $1 \mathrm{~d}$ spin chain for the temperature $T$ of order of the Néel temperature.

On the other hand, for the magnetic field applied along the direction of the chains $b$ the results of the fit are presented in Fig. 3. We used the following set of the parameters $\left(J_{A}^{l} / g \mu_{B}\right)=15 \mathrm{~T},\left(J_{B}^{l} / g \mu_{B}\right)=0.7 \mathrm{~T}$, and $\left(J_{C}^{l} / g \mu_{B}\right)=59 \mathrm{~T}$ (the index $l$ indicates the orientation of the external magnetic field along the chain direction). We also see the very good

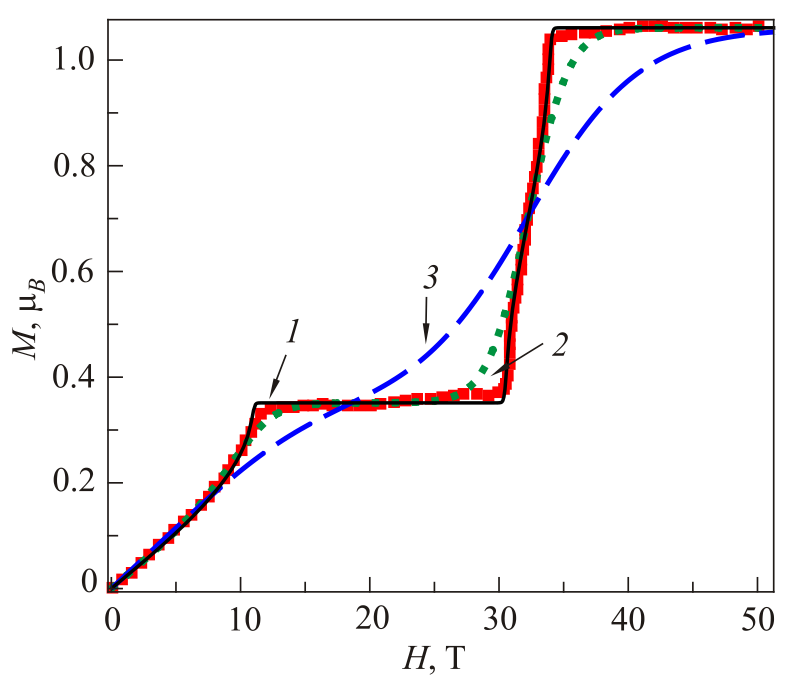

Fig. 2. (Color online) The behavior of the magnetization per site of azurite with the external magnetic field applied perpendicular to $b$ (red squares [7] $T=0.08 \mathrm{~K}$ ) and theoretical calculations for the phenomenological model with the parameters $\left(J_{A}^{p} / g \mu_{B}\right)=11 \mathrm{~T}$, $\left(J_{B}^{p} / g \mu_{B}\right)=3.5 \mathrm{~T}$, and $\left(J_{C}^{p} / g \mu_{B}\right)=64.5 \mathrm{~T}$ and the effective average $g$-factors $g=2.06$ [19]. The black solid line (1) describes the case for $T=0.08 \mathrm{~K}$, the dotted green line (2) describes $T=1.3 \mathrm{~K}$, and the dashed blue line (3) $T=4.2 \mathrm{~K}$.

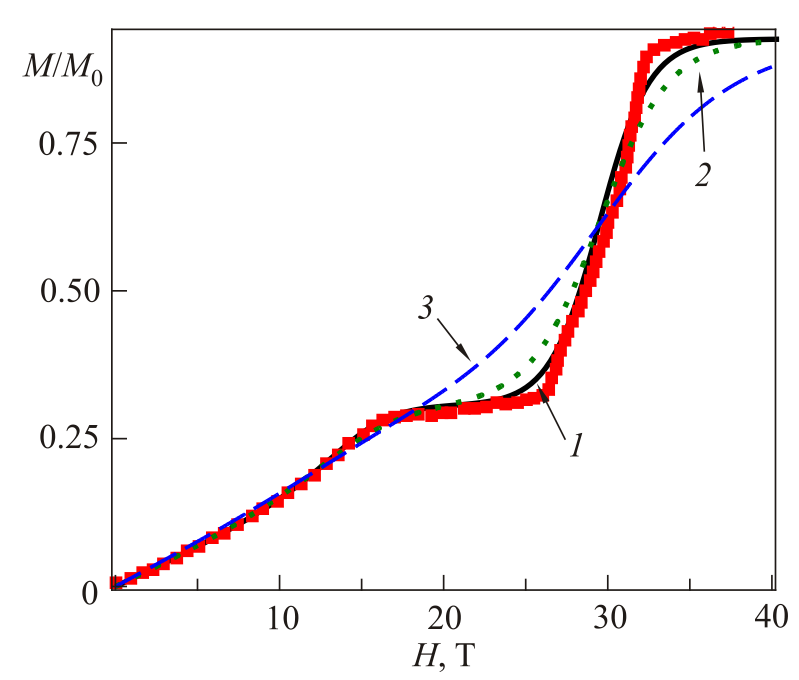

Fig. 3. (Color online) The behavior of the magnetization per site of azurite with the external magnetic field applied parallel to $b$ (red squares [7] $T=1.5 \mathrm{~K}$ ) and theoretical calculations for the phenomenological model with the parameters $\left(J_{A}^{l} / g \mu_{B}\right)=15 \mathrm{~T}$, $\left(J_{B}^{l} / g \mu_{B}\right)=0.7 \mathrm{~T}$, and $\left(J_{C}^{l} / g \mu_{B}\right)=59 \mathrm{~T}$. The black solid line (1) describes the case for $T=1.5 \mathrm{~K}$, the dotted green line (2) describes $T=2 \mathrm{~K}$, and the dashed blue line (3) $T=4.2 \mathrm{~K}$.

agreement of the results of calculations for the phenomenological model and the data of experiments [7]. Different values of the parameters of the model can be considered as follows. In reality there is a magnetic anisotropy in azurite (to remind, the symmetry of its crystal structure is monoclinic, which implies at least a bi-axial magnetic anisotropy; however for the behavior of some characteristics of azurite it is enough to consider only the uniaxial inter-ion anisotropy). Hence, for example, from the values of $J_{A}^{l}$ and $J_{A}^{p}$ we can extract the parameters of the effective isotropic exchange and the magnetic anisotropy $J_{A}=17.9 \mathrm{~K}$ and $J_{A}^{Z}=2,76 \mathrm{~K}$, respectively, which implies that the magnetic anisotropy of that branch is about $15 \%$ of the isotropic exchange. Similar estimations can be obtained for the effective isotropic exchange and magnetic anisotropy of the two other branches of the phenomenological model, yielding the effective isotropic exchange $J_{B}=2.9 \mathrm{~K}$ and $J_{C}=85.2 \mathrm{~K}$ with the effective magnetic anisotropy for the parameters $J_{B}$ and $J_{C}$ equal to $67 \%$ and $4.5 \%$, respectively. Notice that relatively large value of the anisotropy for the parameter $J_{B}^{Z}$ is connected with the larger uncertainty in the definition of the second critical field for azurite for $H \| b$. It is worth mentioning: About twice larger estimates for the values of coupling constants in our approach, comparing with the earlier estimations [10-14], are related to the fact that we use the free fermion model (equivalent to the $X X 0$ spin chain), for which the critical value of the field is $J / g \mu_{B}$, while for the isotropic Heisenberg antiferromagnetic chain, used in [10-14], the critical value of the field is $2 \mathrm{~J} / g \mu_{B}$. Our next step is to calculate the temperature behavior of the magnetic susceptibility of the phenomenological mo- 
del and to compare it with the data of the experiments in azurite

$$
\chi=\frac{\left(g \mu_{B}\right)^{2}}{12 \pi k_{B} T} \sum_{j=1}^{3} \int_{0}^{\pi} d k\left[\cosh \left(\frac{\varepsilon_{k, j}}{2 k_{B} T}\right)\right]^{-2} .
$$

The temperature dependence of the magnetic susceptibility of azurite manifests for $T>T_{N}$ the two-maximum behavior [7], which is supposed to be one of the characteristic features of frustrated magnetic systems [5]. To describe the behavior of the susceptibility for the parameters of the model we use the values obtained for the fitting of the lowtemperature magnetization. The results of such calculations are presented in Fig. 4 for the magnetic field directed along $b$. Here we used the parameters $J_{A}=11.9 \mathrm{~K}, J_{B}=1.05 \mathrm{~K}$ and $J_{C}=88 \mathrm{~K}$, related to the ones in Fig. 3. Notice that we used the renormalization of the parameters (shift of the position by approximately 0.46 and the renormalization of the maximum value by approximately 0.45 ) caused by the difference in the behavior of the magnetic susceptibility of the $X X O$ chain and the $X X Z$ antiferromagnetic spin $1 / 2$ chain calculated in the framework of the exact Bethe ansatz solution using the quantum transfer matrix approach $[2,20]$. We have included to the total susceptibility the contribution from low-temperature part, $T \leq T_{N}$, i.e., the magnetic susceptibility of the magnetically ordered phase (for the experiment in azurite, see Ref. 10), which was calculated in the framework of the mean field approximation. Here we have supposed that the spin chains with the first branch

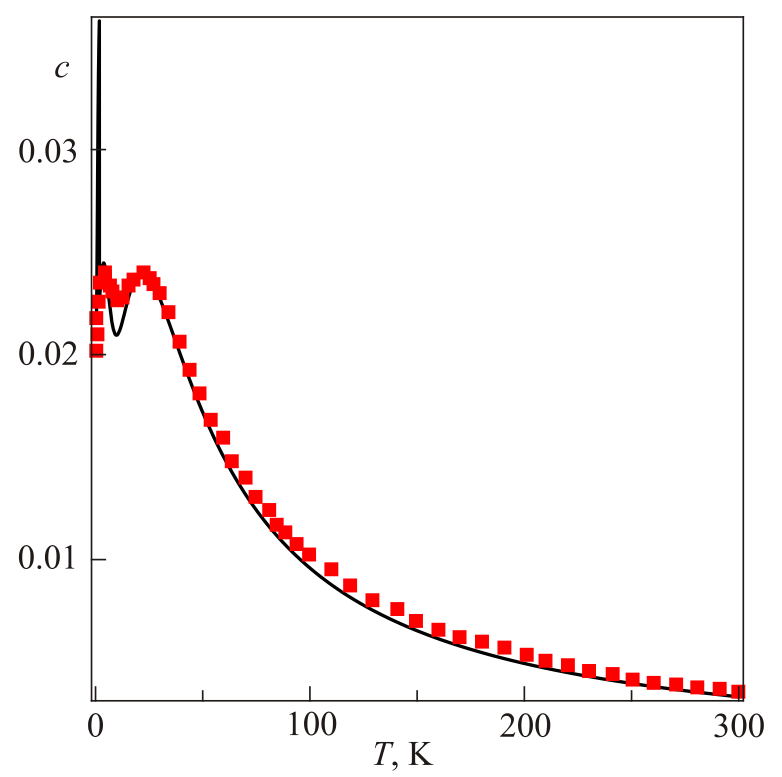

Fig. 4. (Color online) The behavior of the magnetic susceptibility of azurite with the external magnetic field applied parallel to $b$ (red squares [7]) and theoretical calculations for the phenomenological model with the parameters $\left(J_{A}=11.9 \mathrm{~K}, J_{B}=1.05 \mathrm{~K}\right.$, and $J_{C}=88 \mathrm{~K}$ (the solid black line). The low-temperature peak is related to the magnetic ordering at $T_{N}=1.85 \mathrm{~K}$, calculated in the framework of the mean field approximation. spectra are weakly coupled, which produces antiferromagnetic ordering with two magnetic sublattices; on the other hand, the dimerized spin chains with the (gapped) second and the third branches of excitations yield only exponentially small contribution to the ordering and remain effectively one-dimensional at $T \sim T_{N}$. It turns out that the phenomenological model describes very well the twomaximum structure of the temperature dependence of the magnetic susceptibility of azurite at $T>T_{N}$ (it was not reproduced well in the previous calculations of the properties of azurite). The deeper minimum, present in the theoretical result for the magnetic susceptibility, with respect to the data of experiments in azurite $[7,10]$ is related to the free fermion $(X X 0)$ character of our phenomenological model, compared with the more realistic $X X Z$-like behavior $[2,20]$, for which the highs in the temperature behavior of $\chi$ are not so sharp as in the $X X 0$ chain.

Then, in the framework of the phenomenological model we calculate the magnetic contribution to the specific heat

$$
c_{m}=\frac{1}{12 \pi k_{B} T^{2}} \sum_{j=1}^{3} \int_{0}^{\pi} d k \frac{\varepsilon_{k, j}^{2}}{\cosh ^{2}\left(\varepsilon_{k, j} / 2 k_{B} T\right)} .
$$

The results of calculations are presented in Fig. 5 (we used the same set of parameters as in Figs. 3-4). The lowtemperature peak is related to the magnetic ordering at $T_{N}=1.85 \mathrm{~K}$. The contribution for $T \leq T_{N}$ is calculated in the framework of the mean field approximation, in a way similar to the calculation of the contribution to the magnet-

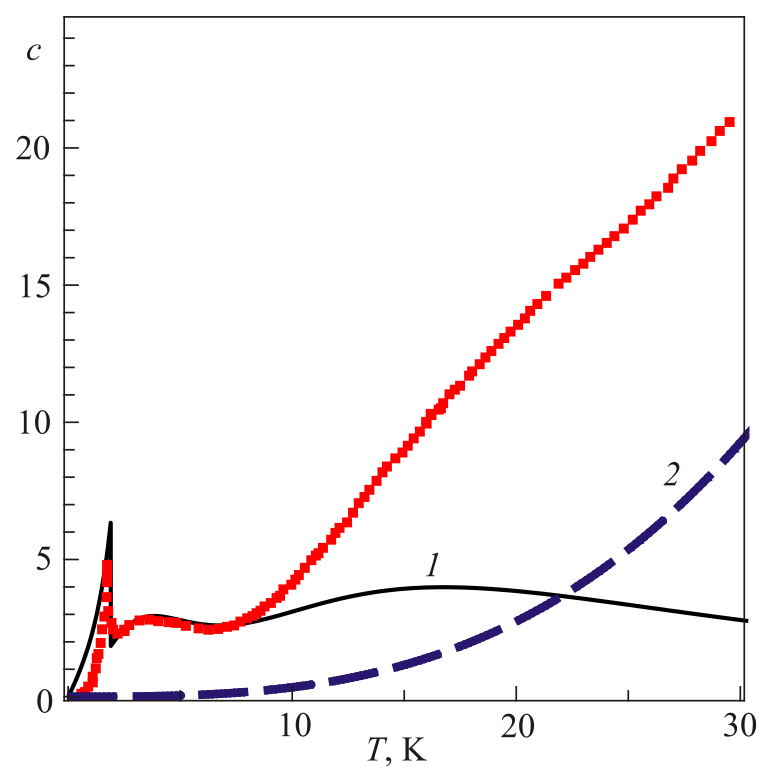

Fig. 5. (Color online) The behavior of the specific heat of azurite at $H=0$ (red squares [7]) and theoretical calculations for the phenomenological model with the same parameters as in Figs. 3 and 4 (the solid black line (1)). The low-temperature peak is related to the magnetic ordering at $T_{N}=1.85 \mathrm{~K}$, calculated in the framework of the mean field approximation. The blue dashed line (2) is the contribution of phonons [10]. 
ic susceptibility, see above. The higher-temperature part of the specific heat for azurite is determined by phonons [10]; the estimation of the contribution of phonons is also shown in Fig. 5. We have also taken into account the difference of the behavior of the specific heat of the phenomenological free fermion model ( $X X 0$ spin chains) with respect to the more realistic $X X Z$ kind, from the comparison of the exact Bethe ansatz results $[2,20]$. We can see that the phenomenological model reproduces well two highs in the temperature dependence (for $T>T_{N}$ ) of the specific heat, related to the contribution of the first and second and third branches of eigenstates of the phenomenological model. Again,we used the renormalization (the position of the maxima, and their values) caused by the difference between the free fermion model (equivalent to $X X 0$ spin $1 / 2$ chain) and more realistic $X X Z$ case. Notice that the weak magnetic field reduces the values of the highs of the magnetic specific heat in our phenomenological model, also in agreement with the data of experiments in azurite [10].

Then, according to [21] it is possible to calculate the temperature dependence of the relative change $\Delta v / v$ of the sound veloclty $v$, caused by the spin-phonon coupling (the exchange striction) in the framework of the phenomenological model. Neglecting the inhomogeneous contributions, we obtain

$$
\frac{\Delta v}{v}=-C_{1} T \chi\left(\chi+C_{2}\right),
$$

where $C_{1}$ and $C_{2}$ are the parameters, related to the gradient of the exchange constants in the spin system (depenent on the direction and the polarization of the sound wave [21]). The results are shown in Fig. 6. One can see that the behavior of the calculated sound velocity is similar to the one, observed in azurite for the temperature behavior of

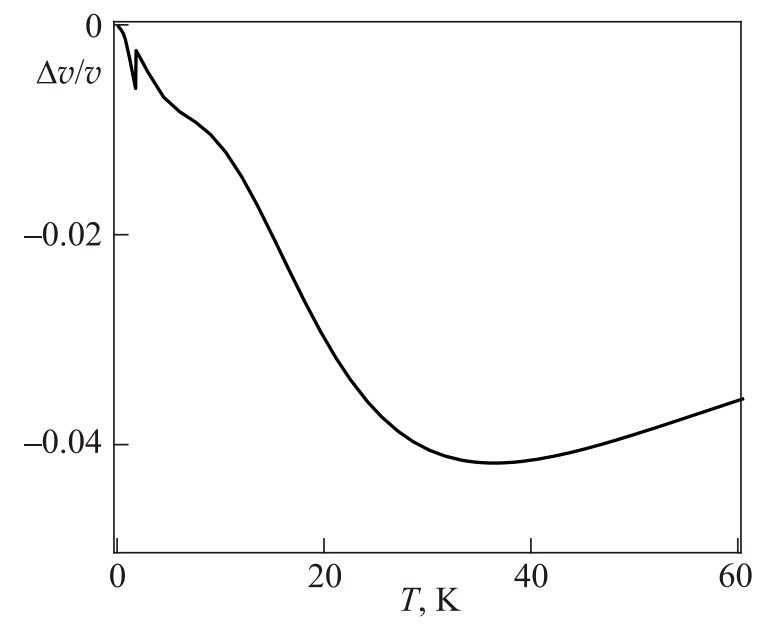

Fig. 6. The temperature dependence of the relative change of the sound velocity at $H=0$ calculated for the phenomenological model with the same parameters as in Figs. 3-5. The low-temperature feature is related to the magnetic ordering at $T_{N}=1.85 \mathrm{~K}$, calculated in the framework of the mean field approximation. the $C_{22}$ elastic modulus [22], connected with the one of $\Delta v / v$.

Finally, in the framework of the phenomenological model we calculate the frequency-magnetic field phase diagram, related to the observed electron spin resonance (ESR) signals in azurite [19,23,24]. The ESR dynamical characteristics of azurite are close to the ones of the neutron scattering $[10,25]$ at $k=0$. The calculations of the resonance frequencies of the ESR in the framework of the phenomenological model for the $1 \mathrm{~d}$ situation for $T>T_{N}$ are performed using the approach similar to Refs. 2, 26. On the other hand, for $T<T_{N}$ we used the standard approach [27] for the magnetically ordered two-sublattice antiferromagnet (to remind, we have supposed that the gapless branch of the phenomenological model plays the major role in the magnetic ordering due to the weak inter-chain coupling, yielding two-sublattice antiferromagnet, see above). The parameters of the exchange and the anisotropy fields for that two-sublattice ordering are extracted from the estimations of the exchange constants and the anisotropy for the magnetic field behavior of the magnetization, see above. The results of calculations are presented in Fig. 7 (we used the values of $g$-factors from the ESR experiments in azurite $[19,23]$ ). We have to distinguish several cases. First, for $H \| b$ the calculations in the framework of the phenomenological theory give two ESR lines (the green long-dashed and dashed-dotted lines), related to the

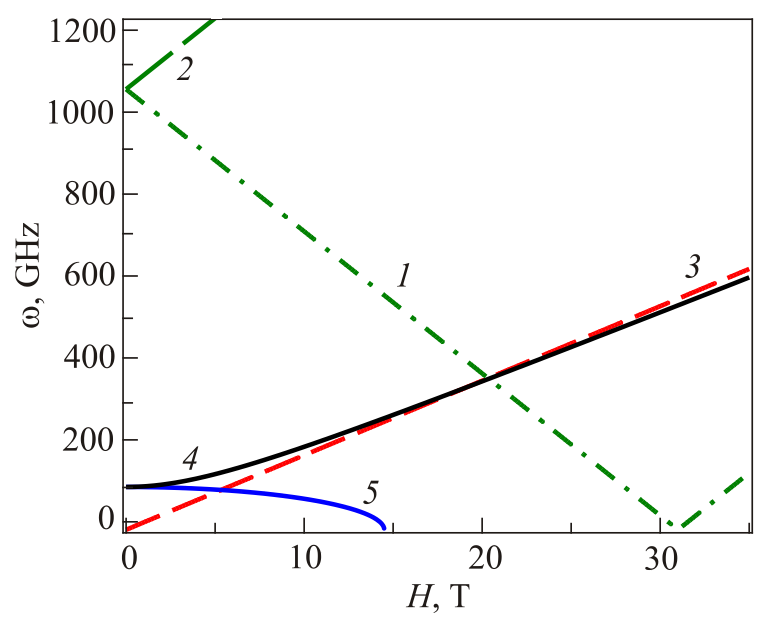

Fig. 7. (Color online) The magnetic field dependence of the resonance frequencies calculated for the phenomenological model with the same parameters as in Figs. 1-6. The green dotteddashed (1) and long-dashed (2) lines correspond to the contribution of the second and the third branches of the phenomenological model. All other lines are connected with the first branch of the model. The red dashed line (3) is related to the ESR signal for $T>T_{N}$ and with the AFMR mode for $T<T_{N}$ (see below). The lines, related to the magnetic ordering at $T<T_{N}$ (the AFMR modes, the black (4) and blue (5) solid lines and the red dashed line), are calculated in the framework of the mean field approximation. 
gapped branches of the phenomenological model. Similar lines were observed in the ESR [19,23,24], as well as in the inelastic neutron scattering [10] both for $T>T_{N}[19,23]$ and for $T<T_{N}[10,24]$. Notice that the experiments [10,19,23,24] observed different slopes (effective $g$-factors in the ESR) for $H<H_{1} \sim 15 \mathrm{~T}$ and for $H>H_{1}$, which was related in [10] to the change of the local mean fields from the antiferromagnetic to the polarized state. The other ESR line (similar line was observed [19,23,24] both for $T>T_{N}$ and for $T<T_{N}$ ) is presented as the dashed red line; it is due to the first branch of the phenomenological model for $T>T_{N}$ (the antiferromagnetic spin chain). For $T<T_{N}$ that ESR line survives as the mode of the antiferromagnetic resonance (AFMR) for the anisotropic two-sublattice magnetic model for the magnetic field, directed perpendicular to the distinguished by the magnetic anisotropy axis. Notice that the weak bi-axial anisotropy, present in azurite, produces a small gap (of order of $30 \mathrm{GHz}$ [23], see also the inelastic neutron scattering results [25]), which is not taken into account in our simplified phenomenological model. However, it is easy to include such a bi-axial anisotropy in the framework of the free fermion model [2]. That small biaxial magnetic anisotropy can slightly renormalize presented above results for other characteristics of azurite; all main features, however remain if that anisotropy is smaller than the uniaxial one. At $T<T_{N}$ our theory also predicts the AFMR mode (the blue solid line), caused by the ordering related to the gapless branch of the phenomenological model. Similar mode was observed in the ESR experiments in azurite [19,23,24]. On the other hand, for the direction of the magnetic field $H$ perpendicular to the $b$ axis, the gapped ESR modes caused by the gapped branches of the phenomenological model (the green long-dashed and dashed-dotted lines) survive (both for $T>T_{N}$ and $T<T_{N}$ ). On the other hand, the gapless branch of the phenomenological model yields the red dashed line in Fig. 7 for $T>T_{N}$, and two AFMR modes, which exist at $T<T_{N}$, shown as the black and blue solid lines in Fig. 7. The line, similar to the upper (black) line was observed in Refs. 19,23,24. Regarding the neutron scattering: In fact the authors of Ref. 25 used the approach, similar to our phenomenological free fermion model (the gapless first branch), to explain theoretically results for the low-energy dynamical structure factor of azurite, which well agree with the observed in experiment data.

In summary, we have proposed the simple phenomenological model to calculate various characteristics of the spin chain system with the geometrical frustration, using azurite as the main example for comparison. The proposed phenomenological model describes very well the behavior of the magnetization, the magnetic susceptibility, the magnetic contribution to the specific heat, the exchange-striction caused renormalization of the sound velocity, and the dynamical characteristics: some features of the ESR and neutron scattering. The advantage of the model is in its simplicity; also it permits to calculate many characteristics of the spin chain compound from the same grounds, without long-time numerical calculations. On the other hand, the model does not take into account bound states, present in spin-1/2 chains with small ratios of the magnetic anisotropy to the isotropic exchange integrals, known from the exact Bethe ansatz results [2]. Notice that we do not discuss the origin of the magnetic anisotropy in azurite: We introduce it by hand, fitting the data, observed in experiments. The symmetry of azurite allows the presence of the Dzyaloshinskii-Moriya interactions, so that the difference in the magnetic behavior parallel and perpendicular to the chain axis $b$ can be caused microscopically by the latter, as well as by the inter-spin anisotropic exchange interactions. We expect that our results can be helpful for experimentalists, who study magnetic properties of azurite. Also, similar models can be constructed for other spin chain materials with possible frustration of spin-spin bonds. For example, our approach (with different values of exchange constants) can be applied to the theoretical description of thermodynamic, magnetic, and dynamic properties of $\mathrm{A}_{3} \mathrm{Cu}_{3} \mathrm{AlO}_{2}\left(\mathrm{SO}_{4}\right)_{4}(\mathrm{~A}=\mathrm{K}, \mathrm{Rb}$, and $\mathrm{Cs})$ [28]. That makes our approach generic, and important for studies of quasione-dimensional quantum spin liquids, especially due to the recent progress in fabrication of new magnetic materials with the properties of spin liquids.

I thank S. Zvyagin and R. Moessner for helpful discussions, and G.A. Zvyagina for the support in production of figures. The support from the Deutsche Forschungsgemeinschaft via SFB1143, and the Institute for Chemistry of the V.N. Karazin Kharkov National University is acknowledged.

1. P.A. Lee, Science 321, 1306 (2008); L. Balents, Nature 464, 199 (2010); L.Savary and L. Balents, Rep. Progr. Phys. 80, 016502 (2017).

2. A.A. Zvyagin, Quantum Theory of One-Dimensional Spin Systems, Cambridge Scientific Publishers, Cambridge (2010).

3. N.D. Mermin and H. Wagner, Phys. Rev. Lett. 17, 1133 (1966).

4. R. Moessner and A.R. Ramires, Phys. Today 59, 24 (2006).

5. For the review of properties of frustrated magnetic systems use, e.g., Frustrated Spin Systems, H.T. Diep (ed.), World Scientific, Singapore (2004); Highly Frustrated Magnetism, C. Lacrois, P. Mendels, and F. Mila (eds.), Springer Berlin (2010); A.A. Zvyagin, Fiz. Nizk. Temp. 39, 1159 (2013) [Low Temp. Phys. 39, 901 (2013)].

6. C. Castelnovo, R. Moessner, and S.L. Sondhi, Nature 451, 42 (2008).

7. H. Kikuchi, Y. Fujii, M. Chiba, S. Mitsudo, T. Idehara, T. Tonegawa, K. Okamoto, T. Sakai, T. Kuwai, and H. Ohta, Phys. Rev. Lett. 94, 227201 (2005); ibid. 97, 089702 (2006); H. Kikuchi, Y. Fujii, M. Chiba, S. Mitsudo, T. Idehara, T. Tonegawa, K. Okamoto, T. Sakai, T. Kuwai, K. Kindo, A. Matsuo, W. Higemoto, K. Nishiyama, M. Horvatić, and C. Bertheir, Progr. Theor. Phys. Suppl. 159, 1 (2005). 
8. H. Kageyama, K. Yoshimura, R. Stern, N.V. Mushnikov, K. Onizuka, M. Kato, K. Kosuge, C.P. Slichter, T. Goto, and Y. Ueda, Phys. Rev. Lett. 82, 3168 (1999); K. Onizuka, H. Kageyama, Y. Narumi, K. Kindo, Y. Ueda, and T. Goto, J. Phys. Soc. Jpn. 69, 1016 (2000); A. Honecker and A. Laüchli, Phys. Rev. B 63, 174407 (2001); K. Kodama, M. Takigawa, M. Horvatić, C. Berthier, H. Kageyama, Y. Ueda, S. Miyahara, F. Becca, and F. Mila, Science 298, 395 (2002); S. Zherlitsyn, V. Tsurkan, A.A. Zvyagin, S. Yasin, S. Erfanifam, R. Beyer, M. Naumann, E. Green, J. Wosnitza, and A. Loidl, Phys. Rev. B 91, 060406(R) (2015).

9. K. Okamoto, T. Tonegawa, Y. Takahashi, and M. Kaburagi, J. Phys.: Condens. Matter 11, 10485 (1999); K. Okamoto, T. Tonegawa, and M. Kaburagi, J. Phys.: Condens. Matter 15, 5979 (2003); T. Sakai, K. Okamoto, and T. Tonegawa, J. Phys.: Conf. Ser. 200, 022052 (2010); A. Miyata, S. Takeyama, and H. Ueda, Phys. Rev. B 87, 214424 (2013).

10. K.C. Rule, A.U.B. Wolter, S. Süllow, D.A. Tennant, A. Brühl, S. Köhler, B. Wolf, M. Lang, and J. Schreuer, Phys. Rev. Lett. 100, 117202 (2008).

11. B. Gu and G. Su, Phys. Rev. Lett. 97, 089701 (2006); Phys. Rev. B 75, 174437 (2007).

12. J. Kang, C. Lee, R.K. Kremer, and M.-H. Whangbo, J. Phys.: Condens. Matter 21, 392201 (2009).

13. A. Honecker, S. Hu, R. Peters, and J. Richter, J. Phys.: Condens. Matter 23, 164211 (2011).

14. H. Jeschke, I. Opahle, H. Kandpal, R. Valentí, H. Das, T. Saha-Dasgupta, O. Janson, H. Rosner, A. Brühl, B. Wolf, M. Lang, J. Richter, S. Hu, X. Wang, R. Peters, T. Pruschke, and A. Honecker, Phys. Rev. Lett. 106, 217201 (2011).

15. E.H. Lieb, T. Schulz, and D. Mattis, Ann. Phys. 16, 417 (1961).

16. M. Yamanaka, M. Oshikawa, and I. Affleck, Phys. Rev. Lett. 79, 1110 (1997).

17. A.A. Zvyagin, Fiz. Tverd. Tela 32, 314 (1990) (in Russian) [Sov. Phys. Solid State 32, 181 (1990)]; A.A. Zvyagin, Phys. Lett. A 158, 333 (1991); A.A. Zvyagin, Fiz. Nizk. Temp. 18, 788 (1992) [Sov. J. Low Temp. Phys. 18, 558 (1992)].

18. T. Giamarchi and A.M. Tsvelik, Phys. Rev. B 59, 11398 (1999).
19. H. Ohta, S. Okubo, T. Kamikawa, T. Kunimoto, Y. Inagaki, H. Kikuchi, T. Saito, M. Azuma, and M. Takano, J. Phys. Soc. Jpn. 72, 2464 (2003).

20. A. Klümper and A.A. Zvyagin, J. Phys.: Condens. Matter 12, 8705 (2000).

21. M. Tachiki and S. Maekawa, Progr. Theor. Phys. 51, 1 (1974); O. Chiatti, A. Sytcheva, J. Wosnitza, S. Zherlitsyn, A.A. Zvyagin, V.S. Zapf, M. Jaime, and A. Paduan-Filho, Phys. Rev. B 78, 094406 (2008); A. Sytcheva, O. Chiatti, J. Wosnitza, S. Zherlitsyn, A.A Zvyagin, R. Coldea, and Z. Tylczynski, Phys. Rev. B 80, 224414 (2009); A.V. Andreev, S. Yasin, Y. Skourski, A.A. Zvyagin, S. Zherlitsyn, and J. Wosnitza, Phys. Rev. B 87, 214409 (2013); S. Erfanifam, S. Zherlitsyn, S. Yasin, Y. Skourski, J. Wosnitza, A.A. Zvyagin, P. McClarty, R. Moessner, G. Balakrishnan, and O.A. Petrenko, Phys. Rev. B 90, 064409 (2014).

22. P.T. Cong, B. Wolf, R.S. Manna, U. Tutsch, M. de Souza, A. Brühl, and M. Lang, Phys. Rev. B 89, 174427 (2014).

23. T. Kamikawa, S. Okubo, T. Kunimoto, H. Ohta, Y. Inagaki, H. Kikuchi, T. Saito, M. Azuma, and M. Takano, Physica B 329-333, 988 (2003); S. Okubo, T. Kamikawa, T. Kunimoto, Y. Inagaki, H. Ohta, H. Nojiri, and H. Kikuchi, J. Magn. Magn. Mater. 272-276, 912 (2004).

24. S. Zvyagin, private communication.

25. K.C. Rule, M. Reehuis, M.C.R. Gibson, B. Ouladdiaf, M.J. Gutmann, J.-U. Hoffmann, S. Gerischer, D.A. Tennant, S. Süllow, and M. Lang, Phys. Rev. B 83, 104401 (2011); K.C. Rule, D.A. Tennant, J.-S. Caux, M.C.R. Gibson, M.T.F. Telling, S. Gerischer, S. Süllow, and M. Lang, Phys. Rev. B 84, 184419 (2011).

26. A.A. Zvyagin, Phys. Rev. B 79, 064422 (2009).

27. C. Kittel, Phys. Rev. 71, 270 (1947); J.H. Van Vleck, ibid. 78, 266 (1950); C. Kittel, ibid. 82, 565 (1951); T. Nagamiya, Progr. Theor. Phys. 6, 350 (1951); F. Keffer and C. Kittel, Phys. Rev. 85, 329 (1952).

28. K. Morita, M. Fujihala, H. Koorikawa, T. Sugimoto, S. Sota, S. Mitsuda, and T. Tohyama, Phys. Rev. B 95, 184412 (2017). 